\title{
Effects of no-reflow phenomenon on ventricular systolic synchrony in patients with acute anterior myocardial infarction after percutaneous coronary intervention
}

\author{
This article was published in the following Dove Press journal: \\ Therapeutics and Clinical Risk Management \\ 24 June 2016 \\ Number of times this article has been viewed
}

\section{Le Wang' \\ Gang Liu' \\ Jun Liu' \\ Mingqi Zheng' \\ Liang $\mathrm{Li}^{2}$}

'Department of Cardiology, The First Hospital of Hebei Medical University, ${ }^{2}$ Department of Cardiology, The Second Hospital of Hebei Medical University, Shijiazhuang, Hebei, People's Republic of China
Correspondence: Liang Li Department of Cardiology, The Second Hospital of Hebei Medical University, No 215, West Heping Road, Shijiazhuang 050000, Hebei, People's Republic of China

Tel +86I8630I0 3882

Email leinll@aliyun.com
Objectives: The aim of this study was to investigate the effect of no-reflow phenomenon on ventricular systolic synchrony via myocardial blush grades (MBGs) in patients with acute anterior myocardial infarction after percutaneous coronary intervention (PCI).

Patients and methods: All patients were divided into two groups and assessed by MBGs. To observe the parameters of the left ventricular function and left ventricular systolic synchrony, equilibrium radionuclide angiography was performed 1 week after PCI and repeated 6 months after acute myocardial infarction (AMI). Measurement data were compared and analyzed by the Student's $t$-test, and the count data were evaluated by the $\chi^{2}$ test. A multivariate regression analysis was performed to assess the contribution of confounding factors.

Results: A total of 100 patients were enrolled in this study: 26 in the no-reflow and 74 in the reflow group. There was no significant difference in terms of age, sex, hypertension history, diabetes history, hyperlipidemia history, and smoking history between the two groups. However, the incidence rate of heart failure with Killip's grade $\geq 2$ in the no-reflow group was significantly higher than that in the reflow group $(38.46 \%$ vs $18.92 \%, P<0.05)$. Six months after the AMI-PCI, the left ventricular ejection fraction, peak ejection rate, and peak filling rate in the no-reflow group were significantly lower than those in the reflow group $(t=2.21,2.29$, and 2.03, $P<0.05$ for all comparisons), but the values of the time to peak ejection rate, time to peak filling rate, phase shift, full width at half maximum, and peak phase standard deviation were all higher ( $t=2.41,2.46,2.00,2.55$, and 2.49, $P<0.05$ for all comparisons), and the incidence rate of major adverse cardiac events in the no-reflow group was also more elevated than the reflow group $\left(53.85 \%\right.$ vs $\left.8.11 \%, \chi^{2}=34.49, P<0.001\right)$.

Conclusion: The no-reflow phenomenon identified by MBGs reflects the no-reperfusion status in the myocardium in the infarction-related zone after AMI. The directly caused reduction in the left ventricular systolic synchrony performance leads to adverse long-term outcomes in patients with AMI.

Keywords: acute anterior myocardial infarction, angioplasty, no-reflow, myocardial blush grades, synchrony

\section{Introduction}

The purpose of the treatment strategy for acute myocardial infarction (AMI) is to rescue the dying heart tissues by completely opening the occluded blood vessels. Thus, once the diagnosis of AMI is made, the initiation of reperfusion therapy with primary percutaneous coronary intervention (PCI) is one of the early and effective management plans. 
However, studies have shown that $>25 \%$ of myocardial tissue blood flow does not completely recover with revascularization, even in areas of the patients body in whom thrombolysis in myocardial infarction (TIMI) flow grade 2 or lower has been achieved at least 10 minutes after the end of the PCI procedure in coronary angiography (CAG) imaging. ${ }^{1-3}$ It has also been reported that for some patients with AMI, after PCI, there still exists poor myocardial reperfusion in blood vessels that have reached TIMI grade 3 blood flow. ${ }^{4}$ Therefore, the value of TIMI grade in accurately assessing myocardial reperfusion after PCI has been put into question. Meanwhile, the use of myocardial blush grades (MBGs) for the assessment of myocardial reperfusion in AMI after PCI has been established as an effective and clinically feasible approach. ${ }^{5,6}$ Nevertheless, the mid- and long-term effects of no-reflow phenomenon after PCI on ventricular systolic synchrony assessed with MBG in patients with AMI have been rarely reported so far. In this study, patients with AMI with no-reperfusion phenomenon after PCI, defined by MBG, were enrolled. The effect of noreflow phenomenon on ventricular systolic synchrony was studied by the application of the computer-assisted equilibrium blood-pool imaging approach.

\section{Patients and methods Study subjects}

A total of 100 patients (67 males and 33 females, aged $62.91 \pm 11.68$ years) were enrolled in this prospective study. They were admitted to The Second Hospital of Hebei Medical University from October 2009 to October 2011 and were treated directly with urgent PCI within 12 hours after the first onset of the symptoms of acute anterior myocardial infarction. The diagnosis of all patients was made in accordance with the World Health Organization (WHO) diagnostic criteria. Inclusion criteria were: 1) first onset of AMI; 2) diagnosis made in accordance with the WHO diagnostic criteria; 3) PCI performed within 12 hours after the onset of symptoms; and 4) voluntary participation. Exclusion criteria were: 1) presence of cerebrovascular accidents or transient ischemic attack history within 6 months; 2) known bleeding history or bleeding tendency; 3 ) a history of major surgery or head trauma within 15 days; 4) aortic dissection; 5) blood pressure $\geq 180 / 90 \mathrm{mmHg}$; 6) severe renal insufficiency; 7) cardiac function Killip's grade 4; and 8) severe arrhythmia or bundle branch block, so that equilibrium radionuclide angiocardiography (ERNA) cannot be performed or the relevant parameter analysis was affected. Written informed consent was obtained from all the participants. This study was approved by the Ethics Committee of Second Hospital of Hebei Medical University.

\section{Methods}

PCI was performed in all patients within 12 hours after the onset of symptoms in accordance with the standard protocol, with residual stenosis $\leq 10 \%$ as the success criterion. CAG images were obtained at 30 frames per second from multiprojection angle using the CAS-330B digital subtraction angiography system (Toshiba, Tokyo, Japan). A complete range of images with the optimal projection angle and effects were selected and assessed by MBG criteria. Angiographic imaging was recorded until the contrast agent (Ultravist ${ }^{\circledR}$ 370, a total of 10-15 mL; Schering, Kenilworth, NJ, USA) filled the coronary vein and flowed back into the coronary sinus. Meanwhile, the slow blood flow caused by coronary artery spasms was excluded through a conventional injection of nitroglycerin $(100 \mu \mathrm{g})$ into the coronary artery. MBG was determined by two experienced physicians using the MBG method: ${ }^{7}$ grade 0 : no contrast agent in the infarction-related zone (IRZ) of the myocardium; grade 1: mild staining with contrast agent of the myocardium in IRZ, but significantly lower than that of the myocardium in the ipsilateral or contralateral non-IRZ; grade 2: moderate staining of the myocardium in IRZ with the contrast agent, but lower than that of the myocardium in the ipsilateral or contralateral non-IRZ; grade 3: normal staining of the myocardium, referring to staining in the IRZ of the myocardium with an extent of the contrast agent identical to that in the ipsilateral or contralateral non-IRZ side. According to the no-reflow MBG standards, patients with an $\mathrm{MBG}$ within the range $0-1$ were classified as the no-reflow group, and as the reflow group when the $\mathrm{MBG}$ was $2-3 .{ }^{7}$ The patients in each group were routinely treated with aspirin, clopidogrel, heparin, nitroglycerin, angiotensinconverting enzyme inhibitor, and $\beta$-blockers.

One week and 6 months after PCI, all patients underwent ERNA using a DST (digital signal technology) dual-head SPECT (single photon emission computed topography) computer system (Sopha, Buc, Cedex, France), and parameters, such as left ventricular systolic function, diastolic function, and synchronization function, were obtained automatically through the software in the computer. The patients were followed up for 6 months for major complications and major cardiovascular events, including postinfarction angina, reinfarction, heart failure, cerebrovascular accident, vital organs bleeding, puncture site hematoma, and death.

\section{Statistical analysis}

ERNA-related parameter measurements were conducted by two nuclear medicine physicians. Statistical analysis was performed using software SPSS 13.0 (SPSS Inc., Chicago, IL, USA). A comparison of measurement data was conducted 
using the Student's $t$-test and of count data by the $\chi^{2}$ test. A multivariate regression analysis was employed to assess the implications of confounding factors. $P<0.05$ was considered statistically significant.

\section{Results}

The flowchart of the study design is illustrated in Figure 1.

\section{Comparison of clinical characteristics}

There were 26 patients in the no-reflow group (17 males and nine females, mean age $63.23 \pm 11.21$ years) and 74 patients in the reflow group (50 males and 24 females, mean age $62.81 \pm 12.27$ years). No significant difference in age, sex, hypertension history, diabetes history, hyperlipidemia history, and smoking history was observed between the two groups. However, in the no-reflow group, the interval between symptom onset and balloon time was significantly longer than that in the reflow group ( $9.06 \pm 5.23$ hours vs $6.76 \pm 4.27$ hours, $P<0.05$ ), and the incidence rate of heart failure with Killip's grade $\geq 2$ in the no-reflow group was also significantly more augmented than that in the reflow group $(38.46 \%$ vs $18.92 \%, P<0.05)$.

\section{Comparison of parameters of cardiac function}

At the 6-month follow-up after AMI-PCI, the values of the cardiac function parameters in the no-reflow group, such as left ventricular ejection fraction, peak ejection rate, and peak filling rate, were significantly lower than those in the reflow group ( $t=2.21,2.29$, and 2.03; $P<0.05$ for all comparisons), but the time to peak ejection rate, time to peak filling rate, phase shift, full width at half maximum, and peak phase standard deviation were higher $(t=2.41$, 2.46, 2.00, 2.55, and 2.49, $P<0.05$ for all comparisons), whereas the incidence rate of major adverse cardiac events (MACEs) in the no-reflow group was more elevated than that in the reflow group $\left(53.85 \%\right.$ vs $8.11 \%, \chi^{2}=34.49$, $P<0.001$; Table 1).

\section{Discussion}

The purpose of our study was to investigate the effect of the no-reflow phenomenon on ventricular systolic synchrony by MBG in patients with AMI after PCI. We found that noreflow identified by MBG reflects the no-reperfusion status in the myocardium in the post-AMI IRZ, causing directly a decrease in left ventricular systolic synchrony performance with adverse long-term outcomes in patients with AMI.

The aim of reperfusion treatment of AMI by PCI is to quickly and fully restore myocardial blood flow to avoid further myocardial necrosis and dysfunction; therefore, the incidence of no-reflow after PCI affects the efficacy of clinical treatment. ${ }^{8} 9$ No-reflow is generally defined as a residual stenosis of $<10 \%$ after responsible coronary stent

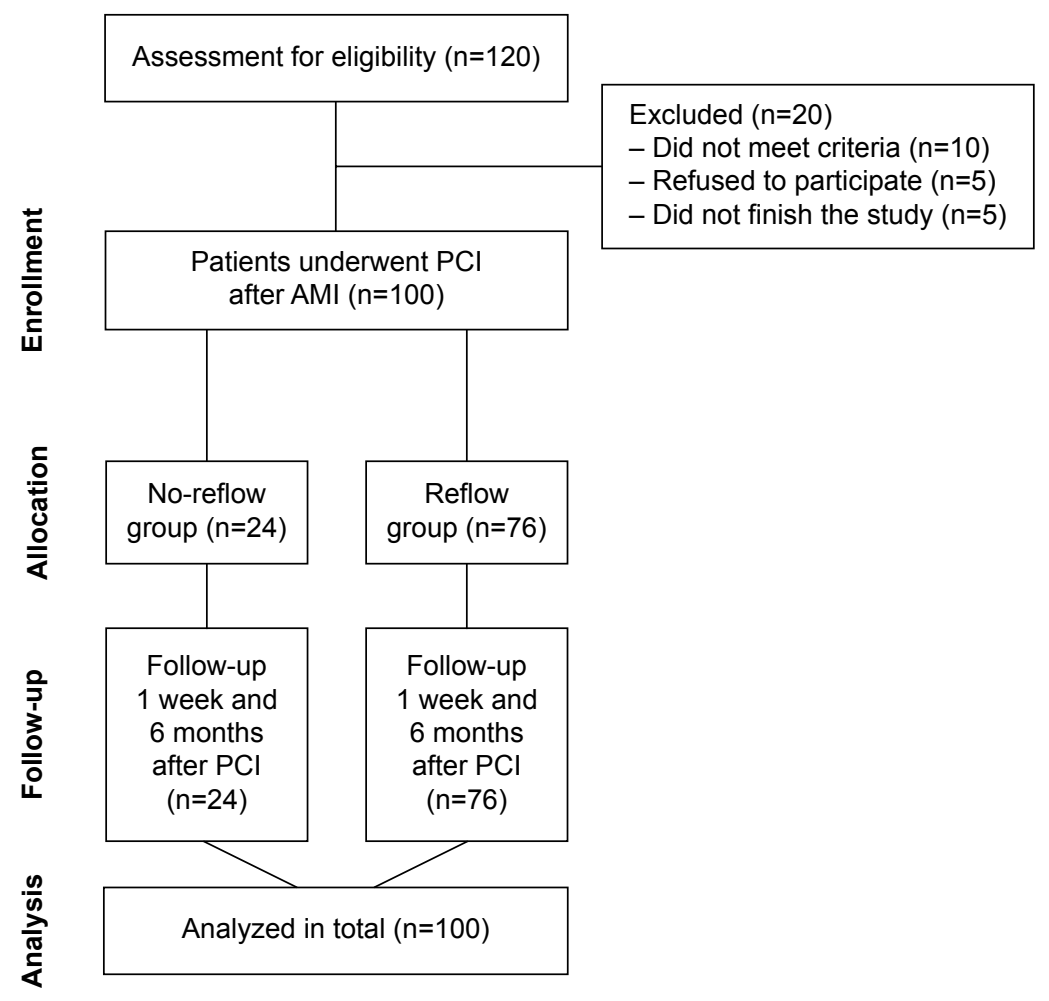

Figure I Flowchart of the study design.

Abbreviations: AMI, acute myocardial infarction; $\mathrm{PCl}$, percutaneous coronary intervention. 
Table I Changes of ERNA parameters in the two groups

\begin{tabular}{|c|c|c|c|c|c|c|c|c|}
\hline & \multicolumn{3}{|l|}{ LVSF } & \multicolumn{2}{|l|}{ LVDF } & \multicolumn{3}{|l|}{ LVSS } \\
\hline & LVEF (\%) & PER (EDV/s) & TPER (ms) & PFR (EDV/s) & TPFR (ms) & PS $\left({ }^{\circ}\right)$ & FWHM $\left(^{\circ}\right)$ & PSD $\left({ }^{\circ}\right)$ \\
\hline \multicolumn{9}{|c|}{ No-reflow (cases: 26) } \\
\hline I week & $28.29 \pm 3.77$ & $1.37 \pm 0.05$ & $211 \pm 25$ & $1.52 \pm 0.23$ & $223 \pm 32$ & $59.99 \pm 20.16$ & $33.88 \pm|0.7|$ & $15.23 \pm 3.15$ \\
\hline 6 months & $36.82 \pm 3.79 \#$ & $1.60 \pm 0.04$ & $179 \pm 22^{\#}$ & $2.05 \pm 0.09^{\#}$ & $186 \pm 16^{\#}$ & $54.68 \pm \mid 6.5 I^{\#}$ & $25.23 \pm 8.37^{\#}$ & $13.57 \pm 3.27$ \\
\hline \multicolumn{9}{|c|}{ Reflow (cases: 74) } \\
\hline I week & $37.89 \pm 3.24 *$ & $1.59 \pm 0.06 *$ & $176 \pm 12^{*}$ & $I .7 I \pm 0.21 *$ & $210 \pm 23 *$ & $51.09 \pm 15.15^{*}$ & $27.03 \pm 6.67^{*}$ & $|3.43 \pm 3.2|$ \\
\hline 6 months & $50.26 \pm 6.17^{* \#}$ & $2.86 \pm 0.09$ *,\# & $150 \pm 16 * \#$ & $2.32 \pm 0.33^{* \# \#}$ & $162 \pm 15^{* \# \#}$ & $41.92 \pm 16.05^{* \#}$ & $19.35 \pm 5.66 * \#$ & $9.24 \pm 1.19^{*, \#}$ \\
\hline
\end{tabular}

Notes: Comparison between no-reflow and reflow groups, $* \mathrm{P}<0.05$. Comparisons were made within each group at two time points after the intervention, I week and 6 months, ${ }^{\#}<<0.05$. Data presented as mean \pm standard error.

Abbreviations: EDV, end diastolic volume; ERNA, equilibrium radionuclide angiocardiography; FWHM, full width at half maximum; LVDF, left ventricular diastolic function; LVEF, left ventricular ejection fraction; LVSS, left ventricular systolic synchrony; LVSF, left ventricular systolic function; PER, peak ejection rate; PFR, peak filling rate; PS, phase shift; PSD, phase standard deviation; TPER, time to PER; TPFR, time to peak filling rate.

implantation with no vascular dissection and little to no advanced blood perfusion into the myocardium. ${ }^{10}$ As a complication, no-reflow can lead to blood pressure decreases and cardiogenic shock as well as increased in-hospital mortality and re-myocardial infarction. ${ }^{11}$ No-reflow is also a predictor of continued ischemia, infarction extension, ventricular remodeling, and cardiac dysfunction as well as a marker of serious cardiac and microvascular injury. ${ }^{12}$ Currently, angiography is the most common and valuable method for detecting no-reflow. ${ }^{13}$ However, studies have shown that the use of MBG is an effective and clinically feasible approach for assessing myocardial reperfusion in AMI after $\mathrm{PCI},{ }^{5,6}$ and our results confirmed this finding.

TIMI grade 3 blood flow has been revealed to indicate only that the velocity of blood flow in the large epicardial vessels and branches has reached a normal level. However, it did not reflect the state of perfusion of the small coronary artery. ${ }^{14,15}$ Therefore, a no-reflow process exists even in the IRZ of the myocardium, whose blood was supplied by blood vessels that underwent PCI and reached TIMI grade 3 blood flow, indicating that TIMI grade 3 blood flow does not fully reflect the reperfusion status of the IRZ of the myocardium after successful opening of the supplying blood vessels. ${ }^{4}$ This phenomenon has been confirmed by positron emission tomography, myocardial contrast echocardiography, and ${ }^{99 m}$ technetium-methoxyisobutylisonitrile myocardial perfusion SPECT imaging of the infarct zone. ${ }^{16,17}$ Currently, it is believed that the status of poor reperfusion or its absence, which can lead to the so-called "no-reflow phenomenon", is caused by damages to the integrity and permeability of the micro-vessels during ischemia and reperfusion. Under the background of interventional therapy, its mechanism also includes blockage of distal blood vessels by debris of the plaque. ${ }^{4,5,7,14,18}$ Stone et al ${ }^{19}$ speculated that the MBG system was more accurate than the TIMI grade system and was also closely associated with MACE. Meanwhile, MBG is an independent predictor of MACE in hospital settings. With the improvement of image quality and resolution of CAG, MBG has achieved a broad clinical application.

Some reports ${ }^{18,20}$ suggest that regardless of the size of the infarct, adequate blood flow in the IRZ could reduce the ventricular volume at a later post-cardiac infarction stage, and the blood flow velocity in the IRZ also affects the procedure of ventricular remodeling. In chronic ischemia, abnormalities in the synthesis and degradation of myocardial collagen matrix could cause a decrease in the production of collagen, ${ }^{21}$ promote ventricular remodeling, and ultimately lead to ventricular dilation in no-reflow patients. In the current study, through ERNA, we accurately and directly compared the cardiac functions of the two groups and quantitatively analyzed the changes in ventricular systolic synchrony using radionuclide phase analysis. After AMI, the contractibility of the infarcted myocardium was reduced, no movement was detected, and no contradictory ventricular wall motion was manifested. To compensate for the abnormal systolic function in the IRZ and to maintain normal cardiac output, the contraction of the non-infarcted myocardium was increased, resulting in a loss of coordination of contraction/dilation of the adjacent myocardium and causing a shift of the local relevant phase and delay of the contraction phase. With time, scars will be formed in the infarct zone, myocardial collagen will be deposited in the non-infarct zone, myocardial stiffness will increase, and ventricular systolic synchrony will decline further.

In this article, we report our ERNA results showing that PCI performed after AMI in the patients in the reflow group was therapeutically effective and resulted in achieving more timely and long-lasting beneficial effects, such as an improvement in hemodynamics, recovery of ventricular wall movement, and inhibition of ventricular remodeling. In the group of patients with reflow, the effective and sustained opening of 
IRZ decreased the preload and afterload of the heart, reduced infarct size, and improved the ventricular contraction/dilation function in the acute phase after AMI. It was also favorable for the coordination of contraction/dilation in various parts of the ventricle and the left ventricular systolic synchrony, as well as for the overall ventricular ejection effect, serving as an effective repressor of ventricular remodeling. Furthermore, over time, this effect became more pronounced.

A recent discussion on the emerging bioresorbable vascular scaffolds (BVS) is also noteworthy. ${ }^{22}$ Compared with the conventional metal stents, BVS have many advantages, including the maintenance of the normal physiological and anatomic structure of the stented blood vessel, the significant decrease in inflammation compared to that caused by the use of conventional stents, and their eventual absorption and achievement of a normal vasomotor tone. Our future plan is to study how BVS affect the no-reflow phenomenon observed after the utilization of conventional stents and to elucidate the underlying mechanism.

There are a few potential limitations of this current study. First of all, the number of study subjects was small; we managed to recruit a total of only 100 patients, and there were only 26 cases in the no-reflow group. Second, the follow-up time (only 6 months) was not sufficiently long to observe the overall lasting impacts; a longer follow-up period is needed to assess the long-term effect of the intervention. We plan to recruit more patients in future studies, and we are currently still following the patients included in this study, and additional results will be published in the future.

\section{Conclusion}

The no-reflow phenomenon determined by MBG after direct PCI reflects the status of a loss of reperfusion in the IRZ of the myocardium, which leads to a decrease in the left ventricular systolic function and synchronization, and directly affects the long-term prognosis in patients with AMI.

\section{Disclosure}

The authors report no conflicts of interest in this work.

\section{References}

1. Tanaka A, Kawarabayashi T, Nishibori Y, et al. No-reflow phenomenon and lesion morphology in patients with acute myocardial infarction. Circulation. 2002;105(18):2148-2152.

2. Maekawa Y, Asakura Y, Anzai T, et al. Relation of stent overexpansion to the angiographic no-reflow phenomenon in intravascular ultrasoundguided stent implantation for acute myocardial infarction. Heart Vessels. 2005;20(1):13-18.

3. Safi AM, Kwan TW. "No-reflow" phenomenon following percutaneous coronary intervention: an uncommon complication. Angiology. 2000;51(3):247-252.
4. Fazel R, Krumholz HM, Bates ER, et al; National Registry of Myocardial Infarction (NRMI) Investigators. Choice of reperfusion strategy at hospitals with primary percutaneous coronary intervention: a National Registry of Myocardial Infarction analysis. Circulation. 2009;120(24):2455-2461.

5. Esposito G, Dellegrottaglie S, Chiariello M. The extent of irreversible myocardial damage and the potential for left ventricular repair after primary percutaneous coronary intervention. Am Heart J. 2010; 160(6 suppl):S4-S10.

6. Ernande L, Cachin F, Chabrot P, et al. Rest and low-dose dobutamine Tc-99m-mibi gated-SPECT for early prediction of left ventricular remodeling after a first reperfused myocardial infarction. J Nucl Cardiol. 2009;16(4):597-604.

7. Brzezinska B, Loboz-Grudzien K, Sokalski L. Patterns of post-MI left ventricular volume changes - clinical implications. Kardiol Pol. 2007;65(10):1190-1198.

8. Montalescot G, van 't Hof AW, Lapostolle F, et al. Prehospital ticagrelor in ST-segment elevation myocardial infarction. $N$ Engl J Med. 2014;371(11):1016-1027.

9. Waltenberger J, Gelissen M, Bekkers SC, et al. Clinical pacing postconditioning during revascularization after AMI. JACC Cardiovasc Imaging. 2014;7(6):620-626.

10. Wang J, Liu H, Salerno TA, et al. Does normothermic normokalemic simultaneous antegrade/retrograde perfusion improve myocardial oxygenation and energy metabolism for hypertrophied hearts? Ann Thorac Surg. 2007;83(5):1751-1758.

11. Harrison RW, Aggarwal A, Ou FS, et al; American College of Cardiology National Cardiovascular Data Registry. Incidence and outcomes of no-reflow phenomenon during percutaneous coronary intervention among patients with acute myocardial infarction. Am J Cardiol. 2013; 111(2):178-184.

12. Akpek M, Kaya MG, Lam YY, et al. Relation of neutrophil/lymphocyte ratio to coronary flow to in-hospital major adverse cardiac events in patients with ST-elevated myocardial infarction undergoing primary coronary intervention. Am J Cardiol. 2012;110(5):621-627.

13. Hou Y, Ma Y, Fan W, et al. Diagnostic accuracy of low-dose 256-slice multi-detector coronary CT angiography using iterative reconstruction in patients with suspected coronary artery disease. Eur Radiol. 2014; 24(1):3-11.

14. Cortina A, Ambrose JA, Prieto-Granada J, et al. Left ventricular function after myocardial infarction: clinical and angiographic correlations. J Am Coll Cardiol. 1985;5(3):619-624.

15. Shih H, Lee B, Lee RJ, Boyle AJ. The aging heart and post-infarction left ventricular remodeling. J Am Coll Cardiol. 2011;57(1):9-17.

16. Greaves K, Dixon SR, Fejka M, et al. Myocardial contrast echocardiography is superior to other known modalities for assessing myocardial reperfusion after acute myocardial infarction. Heart. 2003;89(2):139-144.

17. Visser CA. Left ventricular remodelling after myocardial infarction: importance of residual myocardial viability and ischaemia. Heart. 2003;89(10):1121-1122.

18. Mazzadi AN, Andre-Fouet X, Costes N, Croisille P, Revel D, Janier MF. Mechanisms leading to reversible mechanical dysfunction in severe CAD: alternatives to myocardial stunning. Am J Physiol Heart Circ Physiol. 2006;291(6):H2570-H2582.

19. Stone GW, Peterson MA, Lansky AJ, Dangas G, Mehran R, Leon MB. Impact of normalized myocardial perfusion after successful angioplasty in acute myocardial infarction. J Am Coll Cardiol. 2002;39(4): 591-597.

20. Parodi G, Memisha G, Carrabba N, et al. Prevalence, predictors, time course, and long-term clinical implications of left ventricular functional recovery after mechanical reperfusion for acute myocardial infarction. Am J Cardiol. 2007;100(12):1718-1722.

21. Jugdutt BI. Ventricular remodeling after infarction and the extracellular collagen matrix: when is enough enough? Circulation. 2003; 108(11):1395-1403.

22. Devito F, Zito A, Dachille A, et al. Bioresorbable vascular scaffolds: design, clinical trials, and current applications. Coron Artery Dis. 2016; 27(2):151-158. 


\section{Publish your work in this journal}

Therapeutics and Clinical Risk Management is an international, peerreviewed journal of clinical therapeutics and risk management, focusing on concise rapid reporting of clinical studies in all therapeutic areas, outcomes, safety, and programs for the effective, safe, and sustained use of medicines. This journal is indexed on PubMed Central, CAS,

EMBase, Scopus and the Elsevier Bibliographic databases. The manuscript management system is completely online and includes a very quick and fair peer-review system, which is all easy to use. Visit $\mathrm{http}: / / \mathrm{www}$.dovepress.com/testimonials.php to read real quotes from published authors.

Submit your manuscript here: http://www.dovepress.com/therapeutics-and-clinical-risk-management-journal 